\title{
Nanoporous Glass Integrated in Volumetric Bar-Chart Chip for Point-of-Care Diagnostics of Non-Small Cell Lung Cancer
}

\author{
Ying Li ${ }^{\dagger, \ddagger}$, Jie Xuan ${ }^{\S}$, Yujun Song ${ }^{\dagger, \ddagger}$, Wenjin $\mathrm{Qi}^{\perp, \dagger}$, Bangshun $\mathrm{He}^{\Gamma, \dagger}$, Ping Wang ${ }^{\star}$, , and \\ Lidong Qin ${ }^{*},+\neq$ \\ †Department of Nanomedicine, Houston Methodist Research Institute, 6670 Bertner Avenue, \\ Houston, Texas 77030, United States \\ §Department of Pathology and Genomic Medicine, Houston Methodist Hospital, 6670 Bertner \\ Avenue, Houston, Texas 77030, United States \\ ‡Department of Cell and Developmental Biology, Weill Medical College of Cornell University, New \\ York, New York 10065, United States \\ ${ }^{\perp}$ Department of Obstetrics \& Gynecology, The First Affiliated Hospital of Kunming Medical \\ University, Kunming 650032, China \\ ${ }^{\Gamma}$ Central Laboratory, Nanjing First Hospital, Nanjing Medical University, Nanjing 210006, China
}

\section{Abstract}

Point-of-care (POC) testing has the potential to enable rapid, low-cost, and large-scale screening. POC detection of a multiplexed biomarker panel can facilitate the early diagnosis of non-small cell lung cancer (NSCLC) and, thus, may allow for more timely surgical intervention for life-saving treatment. Herein, we report the nanoporous glass (NPG) integrated volumetric bar-chart chip (VChip) for POC detection of the three NSCLC biomarkers CEA, CYFRA 21-1, and SCCA, by the naked eye. The 3D nanostructures in the NPG membrane efficiently increase the number of binding sites for antibodies and decrease the diffusion distance between antibody and antigen, enabling the low detection limit and rapid analysis time of the NPG-V-Chip. We utilized the NPGV-Chip to test the NSCLC biomarker panel and found that the limit of detection can reach 50 $\mathrm{pg} / \mathrm{mL}$ (10-fold improvement over the original V-Chip), and the total assay time can be decreased from 4 to $0.5 \mathrm{~h}$. We then detected CEA in 21 serum samples from patients with common cancers, and the on-chip results showed good correlation with the clinical results. We further assayed 10 lung cancer samples using the device and confirmed the results obtained using conventional ELISA methods. In summary, the NPG-V-Chip platform has the ability of multiplex, low detection limit, low cost, lack of need for accessory equipment, and rapid analysis time, which may render the V-Chip a useful platform for quantitative POC detection in resource-limited settings and personalized diagnostics.

\footnotetext{
“Corresponding Authors: 1qin@ houstonmethodist.org. pwang@ houstonmethodist.org. Supporting Information

The Supporting Information is available free of charge on the ACS Publications website at DOI: 10.1021/acsnano.5b07357. Additional materials, methods, figures, and tables (PDF)

Notes

The authors declare no competing financial interest.
} 


\section{Graphical abstract}

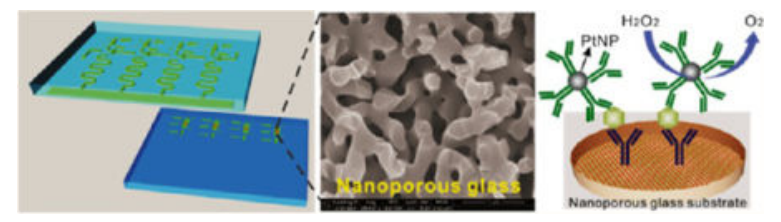

\section{Keywords}

nanoporous glass; point-of-care testing; NSCLC; biomarker detection; microfluidics; V-Chip

Biomarkers play a critical role in the screening, diagnosis, and therapeutic monitoring of disease. ${ }^{1-5}$ Detection and quantification of biomarkers via point-of-care (POC) testing has the potential to carry out these processes more efficiently than conventional methods. ${ }^{6-8}$

Lung cancer is currently the leading cause of cancer-related deaths in the United States, and approximately $80 \%$ of lung cancer cases are non-small cell lung cancer (NSCLC). ${ }^{9-12}$ POC diagnosis of NSCLC provides a means to catch the disease at an early stage and may allow for more timely surgical intervention and further improvement of the survival rate. ${ }^{13,14}$ It has been reported that the optimal combination of serum tumor biomarkers for NSCLC is carcinoembryonic antigen (CEA), squamous cell carcinoma antigen (SCCA), and cytokeratin 19 fragment (CYFRA 21-1). ${ }^{11,14}$ All three biomarkers have low cutoff values $(\sim 1 \mathrm{ng} / \mathrm{mL})$ and can be present in the serum at a wide range of concentrations ( 3 orders of magnitude ranging from $\sim 100 \mathrm{pg} / \mathrm{mL}$ to $\sim 100 \mathrm{ng} / \mathrm{mL}$ ). ${ }^{11,13}$ Consequently, the ideal POC platform for NSCLC detection should be portable, fast, multiplex, sensitive, specific, and have a wide dynamic range.

Microfluidics is a promising technology for developing POC devices due to its low sample consumption, high integration, portability, and low cost. ${ }^{15-17}$ Many microfluidic-based devices have been developed for immunoassays, ${ }^{16,18-20}$ these include the use of photodiodes for the detection of abused substances, ${ }^{21}$ application of a portable surface plasmon resonance system for the measurement of cardiac biomarkers, ${ }^{22}$ utilization of cell phonebased imaging for multiplex detection of ovarian cancer biomarkers, ${ }^{23}$ and employment of fluorescence microscopy to test prostate specific antigen (PSA) for the diagnosis of prostate cancer. $^{24}$ These microfluidic POC platforms demonstrate good performance in terms of sensitivity, multiplexing ability, and dynamic range. However, the majority of these POC methods rely on accessory instruments for quantitative readouts, hindering their broad use in clinical settings and personalized diagnostics. To develop a truly integrated POC platform, our group reported a microfluidic-based volumetric bar-chart chip (V-Chip). A V-Chip is a completely stand-alone microfluidic device that enables low cost, high portability, multiplexing, and naked-eye detection. Our previous work has demonstrated the availability of V-Chip for visual quantification of biomolecules, including protein biomarkers, ${ }^{15,20}$ DNA, ${ }^{25}$ and abused substances. ${ }^{19}$ However, the original V-Chip design has its limitations when applied to NSCLC diagnosis; sensitivity is not sufficiently low $(\sim 0.5 \mathrm{ng} / \mathrm{mL}$, ideally $0.1 \mathrm{ng} / \mathrm{mL})$ and the assay time is relatively long $(\sim 4 \mathrm{~h}) .{ }^{15,20}$ Thus, it remains a challenge to develop a truly integrated platform with high sensitivity and rapid analysis time. 
Three dimensional (3D) materials (e.g., sol-gels, nanoporous hydrogels, nanotextured zinc oxide, carbon nanotube) are widely applied in molecular sensing due to the increased effective surface area, which in turn offers a large number of binding sites. ${ }^{26-31}$ In addition, diffusion path length is efficiently decreased for antibodies and antigens in 3D structures. ${ }^{32}$ For instance, nanoporous glasses (NPGs) have proven effective for enzyme immobilization in biocatalysis. ${ }^{33-35}$ However, few reports describing the use of NPG as an ELISA substrate have been found. Here, we integrated 3D NPG membranes into V-Chip and used it for diagnosing NSCLC. The large porosity of the NPG membranes enables antibodies to achieve multipoint attachment and shortens the antigen-antibody diffusion path length. Consequently, the sensitivity of the NPG-V-Chip is lowered to $\sim 50 \mathrm{pg} / \mathrm{mL}$ for the three NSCLC biomarkers and the assay time is reduced to $\sim 0.5 \mathrm{~h}$. We validated the method by measuring of a single marker, CEA, in 21 serum samples from cancer patients, followed by multiplexed quantification of CEA, CYFRA 21-1, and SCCA in lung cancer patients. The on-chip results were consistent with those acquired clinically or using commercial ELISA kits.

\section{RESULTS AND DISCUSSION}

\section{Working Principle}

An NPG-V-Chip consists of two glass slides $(75 \times 50 \times 1 \mathrm{~mm})$, with one as top plate and one as bottom plate. Microstructures on both of the plates were fabricated by using standard photolithography process (Figures S1 and S2). Holes (diameter $=1.6 \mathrm{~mm}$, depth $=0.5 \mathrm{~mm}$; Figure S3a) were made in the center of the ELISA wells, extending halfway through the bottom plate (thickness $=1 \mathrm{~mm}$ ), and the NPG membranes (diameter $=1.5 \mathrm{~mm}$, thickness $=$ $0.4 \mathrm{~mm}$; Figure S3b) were embedded prior to the assembly of the device (Figure 1a). NPG contains abundant nanopores with an average diameter of $200 \mathrm{~nm}$ (Figure S4), which greatly increase the surface area of the membrane (Figure 1b,c).

Each NPG-V-Chip contains four independent assay units. In the loading phase, the reaction wells are connected to the sample inlets and outlets (Figure 1d). Typically, to characterize the device, the ELISA reaction wells are first filled with PtNP solution for an oxygen gas test; $\mathrm{H}_{2} \mathrm{O}_{2}$ is loaded into the reaction wells with smaller well size, and the wells below the $\mathrm{H}_{2} \mathrm{O}_{2}$ wells are kept empty, serving as air spacers and preventing direct contact between samples and ink, which are loaded into the central wells and channels. The serpentine channels in the lower portion of the device are used to read the results. The biomarker assays are based on the standard sandwich ELISA (Figure 1e). PtNPs conjugated to the detection antibodies react with $\mathrm{H}_{2} \mathrm{O}_{2}$ and catalyze the production of oxygen gas. Horizontal sliding of the top plate against the bottom plate causes the ELISA and $\mathrm{H}_{2} \mathrm{O}_{2}$ wells to overlap, and also connects them to the reading channels, where the oxygen gas generated in the ELISA well propels red ink through the serpentine channels to form a bar-chart. The lengths of the ink bars depend on the quantity of oxygen gas released, which corresponds to the amount of antibody-conjugated PtNPs in the ELISA well, and therefore, the concentration of biomarker present in the patient samples (Figure 1f). 


\section{Evaluation of NPG-V-Chip Performance}

We used standard PtNP solutions to evaluate the performance of the NPG-V-Chip. ${ }^{36} \mathrm{PtNPs}$ with an average diameter of $35 \mathrm{~nm}$ were synthesized as previously reported ${ }^{37}$ (Figure S5; Supporting Information). Figure 2a shows that, at the same concentration of $5 \mu \mathrm{g} / \mathrm{mL}$, ink bars of equal length formed in the four reading channels. Next, a series of PtNP solutions at concentrations of $2,4,10$, and $50 \mu \mathrm{g} / \mathrm{mL}$ were used to investigate the relationship between PtNP concentration and ink-bar advancement. Figure $2 \mathrm{~b}$ illustrates the gradient bar-chart corresponding to the varying PtNP concentration. In Figure 2c, the distances advanced by the ink bars in Figure 2a,b are plotted against PtNP concentration. To study the efficacy of NPG, we compared the sensitivity of CEA ELISA on an NPG-V-Chip to that on a 4-plex VChip, which lacks NPG. Here, PtNPs are conjugated to the antibody as ELISA probes. With the use of NGP, each step in the ELISA takes only $5 \mathrm{~min}$ to complete, whereas $1 \mathrm{~h}$ is required to do so without NPG (Table S1). Not only can an assay at the same concentration of $5 \mathrm{ng} / \mathrm{mL}$ CEA be performed in much less time $(0.5 \mathrm{~h}$ on a NPG-V-Chip compared to $4 \mathrm{~h}$ on a V-Chip), the NPG-V-Chip produced more pronounced ink-bar advancement than the original V-Chip, indicating the ability to achieve better sensitivity (Figure 2d,e). We also tested CEA samples at concentrations of $0.05,2,4$, and $10 \mathrm{ng} / \mathrm{mL}$ on the two different types of devices. The quantitative results are shown in Figure 2f. Regardless of concentration, the bar-chart advancements on NPG-based devices were consistently larger than on those without NPG. Notably, it was difficult to obtain a readable signal on the original V-Chip for CEA samples of $0.05 \mathrm{ng} / \mathrm{mL}$, whereas the NPG-V-Chip generated a stable signal. These results clearly demonstrate that integration of NPG elements in a V-Chip improved the assay performance. The abundant nanopores in NPG greatly increased the number of available antibody binding sites, thus enhancing antibody-substrate binding efficiency, and the decreased length of the diffusion path in the interior of the nanostructures accelerated the process of antibody-antigen binding. These two advantages make the NPG-V-Chip ideal for sensitive and rapid detection of biomarkers. Furthermore, we studied the ability of the NPGV-Chip to measure CEA under physiological conditions. Figure $2 \mathrm{f}$ shows that CEA-spiked human serum gave results similar to those obtained with CEA-spiked PBS, indicating that blood components did not substantially interfere with assay performance on the NPG-VChip.

\section{Calibration of NSCLC Biomarkers CEA, CYFRA 21-1, and SCCA}

Calibration curves were acquired for serum spiked with each of the three biomarkers at various concentrations. As shown in Figure $3 \mathrm{a}-\mathrm{c}$, the full response curves ranged from 0.05 to $250 \mathrm{ng} / \mathrm{mL}$ for CEA and SCCA, and 0.05 to $375 \mathrm{ng} / \mathrm{mL}$ for CYFRA 21-1. These three curves were pseudoexponential rather than linear, which may be attributed to the antibodyantigen binding constant, consumption of $\mathrm{H}_{2} \mathrm{O}_{2}$ during the reactions, and other thermodynamic effects. ${ }^{20}$ For all three biomarkers, the signals at $50 \mathrm{pg} / \mathrm{mL}$ were stronger than the background by a factor of at least three standard deviations (SDs), indicating that the detection limit of the assays was at or below this concentration. These data demonstrate the ability of the NPG-V-Chip to assess NSCLC biomarkers. ${ }^{11}$

In addition to calibration, we also examined percent recovery, a factor used to determine assay accuracy, with an acceptable range between $80 \%$ and $120 \% .{ }^{38}$ On the basis of the 
calibration curves, we selected representative concentrations to analyze the three biomarkers $(0.05,25$, and $200 \mathrm{ng} / \mathrm{mL}$ for CEA and SCCA; and $0.05,50$, and $300 \mathrm{ng} / \mathrm{mL}$ for CYFRA 21-1). At all concentrations, percent recovery for all samples was within the desired range, with the exception of one test for CEA $(0.05 \mathrm{ng} / \mathrm{mL})$ and the other test for CYFRA21-1 (50 $\mathrm{ng} / \mathrm{mL}$ ), which showed a percent recovery of $79 \%$ and $73 \%$, respectively (Figure $3 \mathrm{~d}-\mathrm{f}$ ). Barchart images are shown in Figure $3 \mathrm{~g}-\mathrm{i}$. These data confirmed the performance of the biomarker assays.

Next, the specificity of the antibodies for the three biomarkers was tested. On each device from left to right, the first ELISA well served as control, and the second, third, and fourth ELISA wells were coated with anti-CEA, anti-CYFRA21-1, and anti-SCCA antibodies, respectively. All biomarkers were prepared at $25 \mathrm{ng} / \mathrm{mL}$ and only one biomarker was tested on each device. As shown in Figure S6, ink bars advanced only in the reading channel, where the specific antibody was present, and ink bar lengths were in good agreement with the calibration curves. These results suggested that the platform showed good selectivity for the three NSCLC biomarkers.

\section{Detection of CEA in Cancer Patient Serum Samples}

CEA is recognized in clinical diagnostics as a reliable serum biomarker for various types of cancers, including colon and breast cancer. Here, we used the NPG-V-Chip to quantify CEA in serum samples obtained from patients with types of cancer other than lung cancer. Twenty-one serum/plasma samples were collected from the clinical department of Houston Methodist Hospital. After samples were aliquoted, they were loaded directly onto the NPG membranes without further manipulation. Excluding one lane reserved for controls, three samples can be assayed simultaneously per chip. Bar-chart images are shown in Figure 4a-g. Figure 4h,i shows comparisons of clinical results with on-chip readouts $(n=3)$ and demonstrates good correlation between results obtained with the two methods. Patient demographics for these samples are summarized in Table S2. The NPG-V-Chip successfully detected CEA in these 21 patient samples at concentrations ranging from 1.9 to 184.5 $\mathrm{ng} / \mathrm{mL}$, further confirming the wide dynamic range of our method.

\section{POC Detection of NSCLC Markers in Lung Cancer Patient Samples}

With existing methods, more than $50 \%$ of lung cancer patients are not diagnosed until the advanced stages, which makes treatment less likely to succeed and lowers the patients' chances of survival. ${ }^{11}$ Tumor biomarkers are useful for recognizing disease, performing follow-up, maintaining therapeutic control, and even provide information on prognosis. ${ }^{11,13}$ The biomarker panel of CEA, SCCA, and CYFRA 21-1 was proposed for diagnosing NSCLC, which accounts for approximately $80 \%$ of all lung cancer cases. ${ }^{14}$ POC detection of NSCLC biomarkers has the potential to become a useful tool in large-scale population screening and can improve the chances of early diagnosis. ${ }^{39}$

Here, we applied the NPG-V-Chip to detect the three NSCLC biomarkers. As mentioned above, the first unit on the left side of each device was used for control reactions. Moving from left to right, the next three units were used to assay CEA, CYFRA21-1, and SCCA, respectively. The 10 serum/plasma samples collected from lung cancer patients were 
individually assayed directly on the NPG-V-Chip. Each on-chip assay was completed in 30 min. Figure 5a displays the quantification plots for the on-chip results, which correspond to the bar advancements in Figure S7. The biomarker concentrations were obtained from the calibration curves (Figure 5b). The levels of CEA, CYFRA, and SCCA in the patient samples were 1.0-127.5, 0.5-65.5, and 0.35-50.5 ng/mL, respectively. The concentration of CEA in these 10 samples is clinically available (Table S3), whereas CYFRA 21-1 and SCCA concentrations were not provided. We measured CYFRA 21-1 and SCCA using commercial ELISA kits, and the results are displayed in Figure S8 and Table S3. Patient demographics are also summarized in Table S3. The detection ranges of the CYFRA 21-1 and SCCA kits were $0.312-20$ and $0.078-5 \mathrm{ng} / \mathrm{mL}$, respectively. Samples with concentrations outside the kit ranges were diluted and then tested (while the NPG-V-Chip can assay all the samples directly, without any dilution procedures, because of the wide dynamic range). We then used Bland-Altman analysis to examine the correlation between results obtained with the NPGV-Chip and the conventional ELISA method. Figure 5c shows that the two methods agree well with each other, indicating the accuracy and reliability of our device. In summary, these results further demonstrate that our NPG-V-Chip is advantageous for detecting serum biomarkers with regard to sensitivity, dynamic range, and assay time.

\section{CONCLUSIONS}

In this manuscript, we report on the use of the NPG-integrated V-Chip for POC detection of NSCLC biomarkers. The nanostructures in the NPG membrane efficiently increased the surface area available for antibody binding and decreased the diffusion distance for antibody-antigen binding, allowing for a lower detection limit, and reducing the total preparation and analysis time of the NPG-V-Chip. Compared to the previous V-Chip design, the limit of detection was improved by a factor of 10 (down to $\sim 50 \mathrm{pg} / \mathrm{mL}$ ), and the assay time was shortened from $4 \mathrm{~h}$ to $\sim 0.5 \mathrm{~h}$. We applied the NPG-V-Chip to detect CEA in 21 cancer patient serum/plasma samples, as well as CEA, CYFRA21-1, and SCCA in 10 lung cancer patient samples. All on-chip results showed consistent agreement with those acquired clinically or using commercial ELISA kits, demonstrating the reliability of our platform. With the advantages of multiplexing, wide dynamic range, low-cost, low detection limit, and fast assay time, the NPG-V-Chip offers a promising POC tool for clinical settings and personalized diagnosis.

\section{METHODS}

\section{Materials and Chemicals}

Glass slides $(75 \times 50 \times 1 \mathrm{~mm})$ were obtained from Corning Inc. (Corning, NY, USA). SPR 220-7 was obtained from MicroChem Corp. (Newton, MA, USA). MF-CD26 was purchased from Rohm and Haas Electronic Materials (Marlborough, MA, USA). NPG membranes were ordered from boraident $\mathrm{GmbH}$ (Halle, Germany). Hydrogen peroxide $\left(\mathrm{H}_{2} \mathrm{O}_{2} ; 35 \% \mathrm{w} / \mathrm{v}\right.$ in $\mathrm{H}_{2} \mathrm{O}$ ) and silicone oil were purchased from Sigma-Aldrich (St. Louis, MO, USA). Tridecafluoro-1,1,2,2-tetrahydrooctyl-1-trichlorosilane was purchased from Pfaltz and Bauer (Waterbury, CT, USA). Red ink was purchased from Fisher Scientific (Waltham, MA, USA). Amorphous diamond-coated drill bits were purchased from Harvey Tool (Rowley, MA, 
USA). CEA, anti-CEA antibodies, CYFRA 21-1, and anti-CYFRA 21-1 antibodies were obtained from Abcam (Cambridge, U.K.). SCCA was purchased from Antibodies-Online (Atlanta, GA, USA). Anti-SCCA antibodies were purchased from LSBio (Seattle, WA, USA). ELISA kits for analysis of CYFRA 21-1 and SCCA were obtained from Biomatik (Wilmington, DE, USA).

\section{Device Fabrication and Operation}

In our previous publication, we reported on the use of the singleplex V-Chip with a long reading channel to detect biomarkers with a wide dynamic range of concentrations. ${ }^{20}$ Here, we modified the singleplex V-Chip to a 4-plex V-Chip, which allows prolonged ink-barchart movement in a multiplexed fashion. Fabrication of the 4-plex V-Chip was achieved using a standard photolithography process (Figure S1). ${ }^{20}$ Briefly, to fabricate the mold containing the designed wells and channels, SPR220-7 was spun onto glass slides $(75 \times 50 \times$ $1 \mathrm{~mm}$ ) to a thickness of $10 \mu \mathrm{m}$, and exposed to UV light through the transparent mask. Then, the glass slides were immersed in a glass-etching solution to generate the pattern $(\sim 50 \mu \mathrm{m}$ in depth). Sample inlets/outlets were created with a 0.03 -in. diamond drill. To integrate the NPG materials, we drilled holes (diameter $=1.6 \mathrm{~mm}$, depth $=0.5 \mathrm{~mm}$ ) halfway through the thickness $(1 \mathrm{~mm})$ of the bottom plate in the center of the reaction wells, where the NPG membranes (diameter $=1.5 \mathrm{~mm}$, thickness $=0.4 \mathrm{~mm}$ ) can be put inside (Figure S2, S3). Subsequently, the surfaces of the glass slides were treated with plasma and tridecafluoro-1,1,2,2-tetrahydrooctyl-1-trichlorosilane (to render the surfaces hydrophobic), washed with ethanol, and dried with nitrogen gas.

To assemble the device, $3 \mu \mathrm{L}$ of silicone oil was applied to the top plate with the patterns facing up and spread evenly across the surface by gently pressing the top and bottom plates together and sliding them against each other. The oil seal was to prevent leakage when solutions were added. The two plates were then aligned to connect the two reaction wells (the left well for ELISA and the right well for $\mathrm{H}_{2} \mathrm{O}_{2}$ ) to the inlets and outlets. Samples and reagents were loaded via pipetting. To obtain a readout, the top plate was slid horizontally against the bottom, allowing the reaction wells to overlap and connect with the reading channels in the vertical direction. The length of the ink bars, i.e., the distance that the ink was propelled by the oxygen gas, was measured with the rulers on the side at a predetermined reaction time point.

\section{ELISA on the NPG-V-Chip}

Sandwich ELISA on the NPG-V-Chip is performed on the NPG membrane. The membranes are in the shape of a truncated cone (thickness $=0.4 \mathrm{~mm}$ ), with the bottom surface (diameter $=1.5 \mathrm{~mm}$ ) larger than the top surface (diameter $=1.3 \mathrm{~mm}$ ) (Figure S3a). Prior to an assay, the top surface of the NPG was treated with silane, using the method reported previously for the modification of a glass surface (see Supporting Information for details). ${ }^{20}$ Antibodies were covalently immobilized on the surface via reactions between their amino groups and the surface epoxy groups. Capture antibody ( $5 \mu \mathrm{L}$ of $10 \mu \mathrm{g} / \mathrm{mL}$ solution) was added to the membrane, followed by $5 \mathrm{~min}$ incubation at room temperature. The membrane was then washed with PBS containing $0.05 \%$ (v/v) Tween 20 three times and blocked with 5\% (w/v) bovine serum albumin (BSA) for $5 \mathrm{~min}$. Subsequently, $5 \mu \mathrm{L}$ of sample was loaded onto the 
membrane and incubated for $5 \mathrm{~min}$ at room temperature. After that, $5 \mu \mathrm{L}$ of $10 \mu \mathrm{g} / \mathrm{mL}$ PtNPconjugated detection antibody (see Supporting Information for details) was added and the sample was incubated for $5 \mathrm{~min}$, followed by washing. The NPGs were then inserted into the bottom plate, which in turn was juxtaposed to the top plate to assemble the device (Figure S2). Afte $\mathrm{H}_{2} \mathrm{O}_{2}$ and red ink were loaded, the top plate was slid horizontally, initiating the reaction between the antibody-conjugated PtNP probes and $\mathrm{H}_{2} \mathrm{O}_{2}$. PtNPs have been reported to be efficient catalysts of $\mathrm{O}_{2}$ production from $\mathrm{H}_{2} \mathrm{O}_{2}$. The red ink was propelled into the small channels by the oxygen gas generated by this catalytic reaction. Readouts were acquired at the 5 min time point (at room temperature).

\section{Supplementary Material}

Refer to Web version on PubMed Central for supplementary material.

\section{Acknowledgments}

We are grateful for the funding supports from NIH/NIDA 1R01DA035868-01 and Golfers against Cancer Foundation. We thank Dr. Yang Li for the suggestions on device fabrication and method development.

\section{References}

1. Longo G. CANCER BIOMARKERS Detected Twice for Good Measure. Nat Nanotechnol. 2014; 9:959-960. [PubMed: 25466535]

2. Warren AD, Kwong GA, Wood DK, Lin KY, Bhatia SN. Point-of-Care Diagnostics for Noncommunicable Diseases Using Synthetic Urinary Biomarkers and Ppaper Microfluidics. Proc Natl Acad Sci U S A. 2014; 111:3671-3676. [PubMed: 24567404]

3. Zhang B, Kumar RB, Dai H, Feldman BJ. A Plasmonic Chip for Biomarker Discovery and Diagnosis of Type 1 Diabetes. Nat Med. 2014; 20:948-953. [PubMed: 25038825]

4. Zhu G, Ye M, Donovan MJ, Song E, Zhao Z, Tan W. Nucleic Acid Aptamers: an Emerging Frontier in Cancer Therapy. Chem Commun. 2012; 48:10472-80.

5. Shibutani M, Maeda K, Nagahara H, Ohtani H, Sakurai K, Toyokawa T, Kubo N, Tanaka H, Muguruma K, Ohira M, Hirakawa K. Significance of CEA and CA19-9 Combination as a Prognostic Indicator and for Recurrence Monitoring in Patients with Stage II Colorectal Cancer. Anticancer Res. 2014; 34:3753-3758. [PubMed: 24982398]

6. Laksanasopin T, Guo TW, Nayak S, Sridhara AA, Xie S, Olowookere OO, Cadinu P, Meng FX, Chee NH, Kim J, Chin CD, Munyazesa E, Mugwaneza P, Rai AJ, Mugisha V, Castro AR, Steinmiller D, Linder V, Justman JE, Nsanzimana S, et al. A Smartphone Dongle for Diagnosis of Infectious Diseases at the Point of Care. Sci Transl Med. 2015; 7:273re11-273re19.

7. Song YJ, Huang YY, Liu XW, Zhang XJ, Ferrari M, Qin LD. Point-of-Care Technologies for Molecular Diagnostics Using a Drop of Blood. Trends Biotechnol. 2014; 32:132-139. [PubMed: 24525172]

8. Kelley SO, Mirkin CA, Walt DR, Ismagilov RF, Toner M, Sargent EH. Advancing the Speed, Sensitivity and Accuracy of Biomolecular Detection Using Multi-Length-Scale Engineering. Nat Nanotechnol. 2014; 9:969-980. [PubMed: 25466541]

9. Chen Z, Fillmore CM, Hammerman PS, Kim CF, Wong KK. Non-Small-Cell Lung Cancers: a Heterogeneous Set of Diseases. Nat Rev Cancer. 2014; 14:535-546. [PubMed: 25056707]

10. Hammerman PS, Lawrence MS, Voet D, Jing R, Cibulskis K, Sivachenko A, Stojanov P, McKenna A, Lander ES, Gabriel S, Getz G, Sougnez C, Imielinski M, Helman E, Hernandez B, Pho NH, Meyerson M, Chu A, Chun HJE, Mungall AJ, et al. Comprehensive Genomic Characterization of Squamous Cell Lung Cancers. Nature. 2012; 489:519-525. [PubMed: 22960745] 
11. Kulpa J, Wojcik E, Reinfuss M, Kolodziejski L. Carcinoembryonic Antigen, Squamous Cell Carcinoma Antigen, CYFRA 21-1, and Neuron-Specific Enolase in Squamous Cell Lung Cancer Patients. Clin Chem. 2002; 48:1931-1937. [PubMed: 12406978]

12. Takata M, Chikumi H, Matsunami K, Kodani M, Sakamoto T, Hashimoto K, Nakamoto M, Okada K, Kitaura T, Matsumoto S, Kurai J, Yamasaki A, Igishi T, Burioka N, Shimizu E. A New Rapid Method for Detecting Epidermal Growth Factor Receptor Mutations in Non-Small Cell Lung Cancer. Oncol Rep. 2015; 33:1040-1048. [PubMed: 25591975]

13. Chu XY, Hou XB, Song WA, Xue ZQ, Wang B, Zhang LB. Diagnostic Values of SCC, CEA, Cyfra21-1 and NSE for Lung Cancer in Patients with Suspicious Pulmonary Masses A Single Center Analysis. Cancer Biol Ther. 2011; 11:995-1000. [PubMed: 21483235]

14. Moro D, Villemain D, Vuillez JP, Delord CA, Brambilla C. Cea, Cyfra21-1 and Scc in Non-SmallCell Lung-Cancer. Lung Cancer. 1995; 13:169-176. [PubMed: 8581396]

15. Song Y, Xia X, Wu X, Wang P, Qin L. Integration of Platinum Nanoparticles with a Volumetric Bar-Chart Chip for Biomarker Assays. Angew Chem, Int Ed. 2014; 53:1-6.

16. Mok J, Mindrinos MN, Davis RW, Javanmard M. Digital Microfluidic Assay for Protein Detection. Proc Natl Acad Sci U S A. 2014; 111:2110-2115. [PubMed: 24449893]

17. Li Y, Xuan J, Song Y, Wang P, Qin L. A Microfluidic Platform with Digital Readout and UltraLow Detection Limit for Quantitative Ppoint-of-Care Diagnostics. Lab Chip. 2015; 15:3300-3306. [PubMed: 26170154]

18. Gervais L, de Rooij N, Delamarche E. Microfluidic Chips for Point-of-Care Immunodiagnostics. Adv Mater. 2011; 23:H151-H176. [PubMed: 21567479]

19. Li Y, Xuan J, Xia T, Han X, Song Y, Cao Z, Jiang X, Guo Y, Wang P, Qin L. Competitive Volumetric Bar-Chart Chip with Real-Time Internal Control for Point-of-Care Diagnostics. Anal Chem. 2015; 87:3771-3777. [PubMed: 25751686]

20. Song Y, Zhang Y, Bernard PE, Reuben JM, Ueno NT, Arlinghaus RB, Zu Y, Qin L. Multiplexed Volumetric Bar-Chart Chip for Point-of-Care Diagnostics. Nat Commun. 2012; 3:1283-1291. [PubMed: 23250413]

21. Mobini Far HR, Torabi F, Danielsson B, Khayyami M. ELISA on a Microchip with a Photodiode for Detection of Amphetamine in Plasma and Urine. J Anal Toxicol. 2005; 29:790-793. [PubMed: 16356336]

22. Kurita R, Yokota Y, Sato Y, Mizutani F, Niwa O. On-Chip Enzyme Immunoassay of a Cardiac Marker Using a Microfluidic Device Combined with a Portable Surface Plasmon Resonance System. Anal Chem. 2006; 78:5525-5531. [PubMed: 16878891]

23. Wang SQ, Zhao XH, Khimji I, Akbas R, Qiu WL, Edwards D, Cramer DW, Ye B, Demirci U. Integration of Cell Phone Imaging with Microchip ELISA to Detect Ovarian Cancer HE4 Biomarker in Urine at the Point-of-Care. Lab Chip. 2011; 11:3411-3418. [PubMed: 21881677]

24. Okada H, Hosokawa K, Maeda M. Power-Free Microchip Immunoassay of PSA in Human Serum for Point-of-Care Testing. Anal Sci. 2011; 27:237-241. [PubMed: 21415503]

25. Song YJ, Wang YC, Qin LD. A Multistage Volumetric Bar Chart Chip for Visualized Quantification of DNA. J Am Chem. 2013; 135:16785-16788.

26. Jacobs M, Muthukumar S, Selvam AP, Craven JE, Prasad S. Ultra-Sensitive Electrical Immunoassay Biosensors Using Nanotextured Zinc Oxide Thin Films on Printed Circuit Board Platforms. Biosens Bioelectron. 2014; 55:7-13. [PubMed: 24355459]

27. Stroeve P, Ileri N. Biotechnical and Other Applications of Nanoporous Mmembranes. Trends Biotechnol. 2011; 29:259-266. [PubMed: 21388697]

28. Park J, Koh Y, Ko YC, Sohn H. Simply Modified Biosensor for the Detection of Human IgG Based on Protein A-Modified Porous Silicon Interferometer. Bull Korean Chem Soc. 2009; 30:15931597.

29. Niu Z, Zhou W, Chen X, Chen J, Xie S. Highly Compressible and All-Solid-State Supercapacitors Based on Nano-structured Composite Sponge. Adv Mater. 2015; 27:6002-6008. [PubMed: 26316309]

30. Wan P, Wen X, Sun C, Chandran BK, Zhang H, Sun X, Chen X. Flexible Transparent Films Based on Nanocomposite Networks of Polyaniline and Carbon Nanotubes for High-Performance Gas Sensing. Small. 2015; 11:5409-5415. [PubMed: 26294393] 
31. Bai S, Sun C, Yan H, Sun X, Zhang H, Luo L, Lei X, Wan P, Chen X. Healable, Transparent, Room-Temperature Electronic Sensors Based on Carbon Nanotube Network-Coated Polyelectrolyte Multilayers. Small. 2015; 11:5807-5813. [PubMed: 26395971]

32. Kim D, Herr AE. Protein Immobilization Techniques for Microfluidic Assays. Biomicrofluidics. 2013; 7:041501.

33. Yang Z, Williams D, Russell AJ. Synthesis of Protein-Containing Polymers in Organic-Solvents. Biotechnol Bioeng. 1995; 45:10-17. [PubMed: 18623046]

34. Wang P, Dai S, Waezsada SD, Tsao AY, Davison BH. Enzyme Stabilization by Covalent Binding in Nanoporous Sol-Gel Glass for Nonaqueous Biocatalysis. Biotechnol Bioeng. 2001; 74:249-255. [PubMed: 11400098]

35. Kim J, Grate JW, Wang P. Nanostructures for Enzyme Stabilization. Chem Eng Sci. 2006; 61:1017-1026.

36. Du J, Zhu B, Peng X, Chen X. Optical Reading of Contaminants in Aqueous Media Based on Gold Nanoparticles. Small. 2014; 10:3461-3479. [PubMed: 24578321]

37. Zhang T, Fang Q, Wang SL, Qin LF, Wang P, Wu ZY, Fang ZL. Enhancement of Signal-to-Noise Level by Synchronized Dual Wavelength Modulation for Light Emitting Diode Fluorimetry in a Liquid-Core-Waveguide Microfluidic Capillary Electrophoresis System. Talanta. 2005; 68:19-24. [PubMed: 18970279]

38. Castanheira AP, Barbosa AI, Edwards AD, Reis NM. Multiplexed Femtomolar Quantitation of Human Cytokines in a Fluoropolymer Microcapillary film. Analyst. 2015; 140:5609-5618. [PubMed: 26120601]

39. Sakamoto T, Kodani M, Takata M, Chikumi H, Nakamoto M, Nishii-Ito S, Ueda Y, Izumi H, Makino H, Touge H, Takeda K, Yamasaki A, Yanai M, Tanaka N, Igishi T, Shimizu E. A Novel Ppoint-of-Care System for High-Speed Real-Time Polymerase Chain Reaction Testing for Eepidermal Growth Factor Receptor Mutations in Bronchial Lavage Fluids after Transbronchial Biopsy in Patients with Non-Small Cell Lung Cancer. Int J Oncol. 2015; 46:1473-1480. [PubMed: 25651992] 

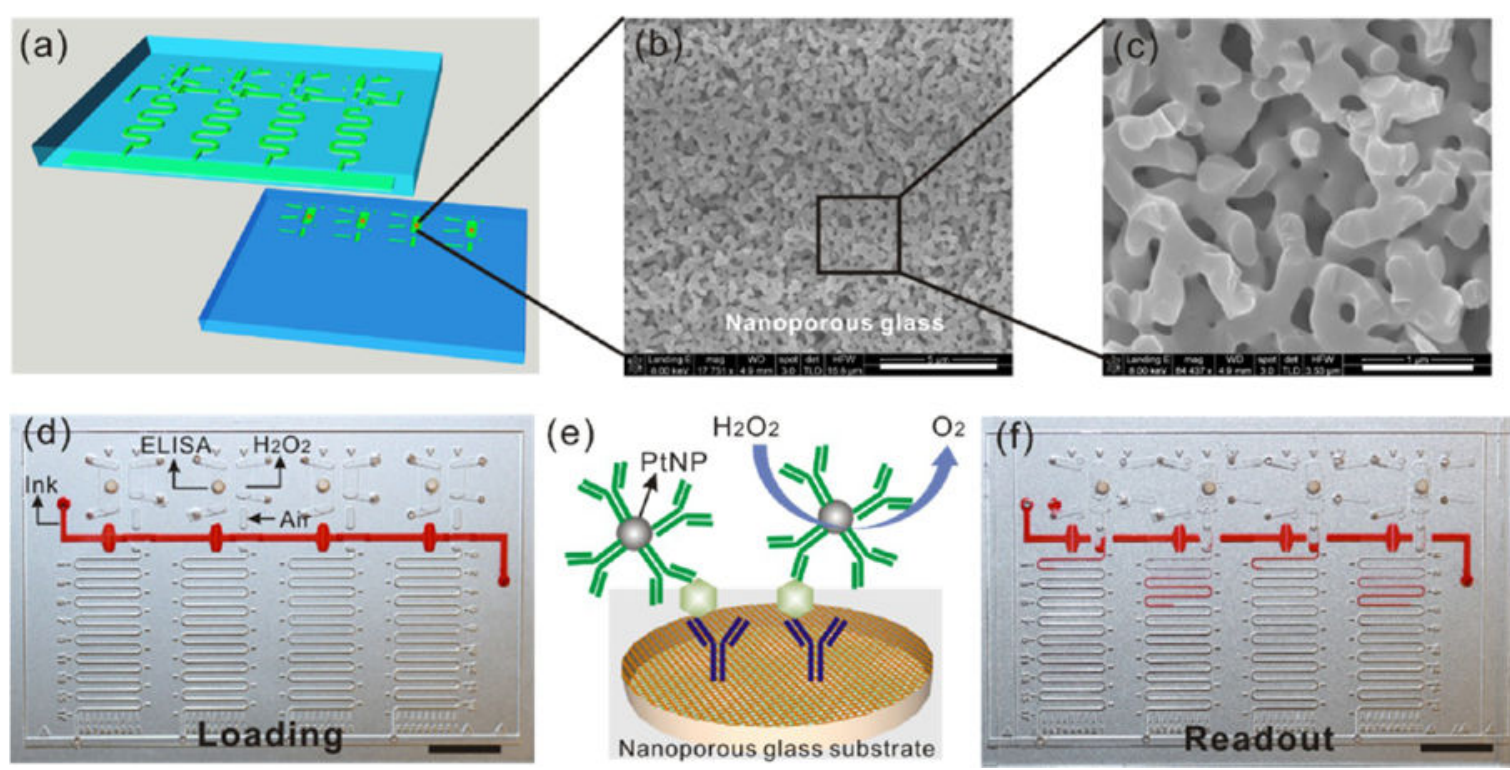

Figure 1.

Working principle of the NPG-V-Chip. (a) Schematic diagram of the unassembled device, which is composed of a top and bottom plate. The 3D nanoporous glass (NPG) membrane (orange cylinder) is inserted into the hole made in the bottom plate. (b and c) SEM images of the NPG, with scale bar representing $5 \mu \mathrm{m}$ (b) and $1 \mu \mathrm{m}$ (c). The NPG is a 3D structure and has abundant nanopores ( $200 \mathrm{~nm}$ diameter). The porous structure provides a large surface area, which should increase the binding efficiency of antibodies. (d) Loading phase of the device. ELISA is performed on the NPG (white structure) embedded in the well; $\mathrm{H}_{2} \mathrm{O}_{2}$ is loaded in the well adjacent to the NPG well; the well below the $\mathrm{H}_{2} \mathrm{O}_{2}$ well is empty, and ink is loaded into the central channel, which extends from left to right. (e) Principle of biomarker detection based on sandwich ELISA on the NPG. PtNPs are used as the ELISA probes and catalyze generation of oxygen from $\mathrm{H}_{2} \mathrm{O}_{2}$. The amount of oxygen generated reflects the concentration of the target biomarker. (f) Readout phase of the device.

Horizontal sliding of the top plate from right to left causes the ELISA and $\mathrm{H}_{2} \mathrm{O}_{2}$ wells to overlap, and also connects the wells and channels in the vertical direction. Oxygen generated from the PtNP- $\mathrm{H}_{2} \mathrm{O}_{2}$ reaction will propel the red ink forward in the serpentine channels. The readout (i.e., distance traveled by the red ink) is obtained after a specified time, typically 5 min. Scale bar, $1 \mathrm{~cm}$ for (d) and (f). 

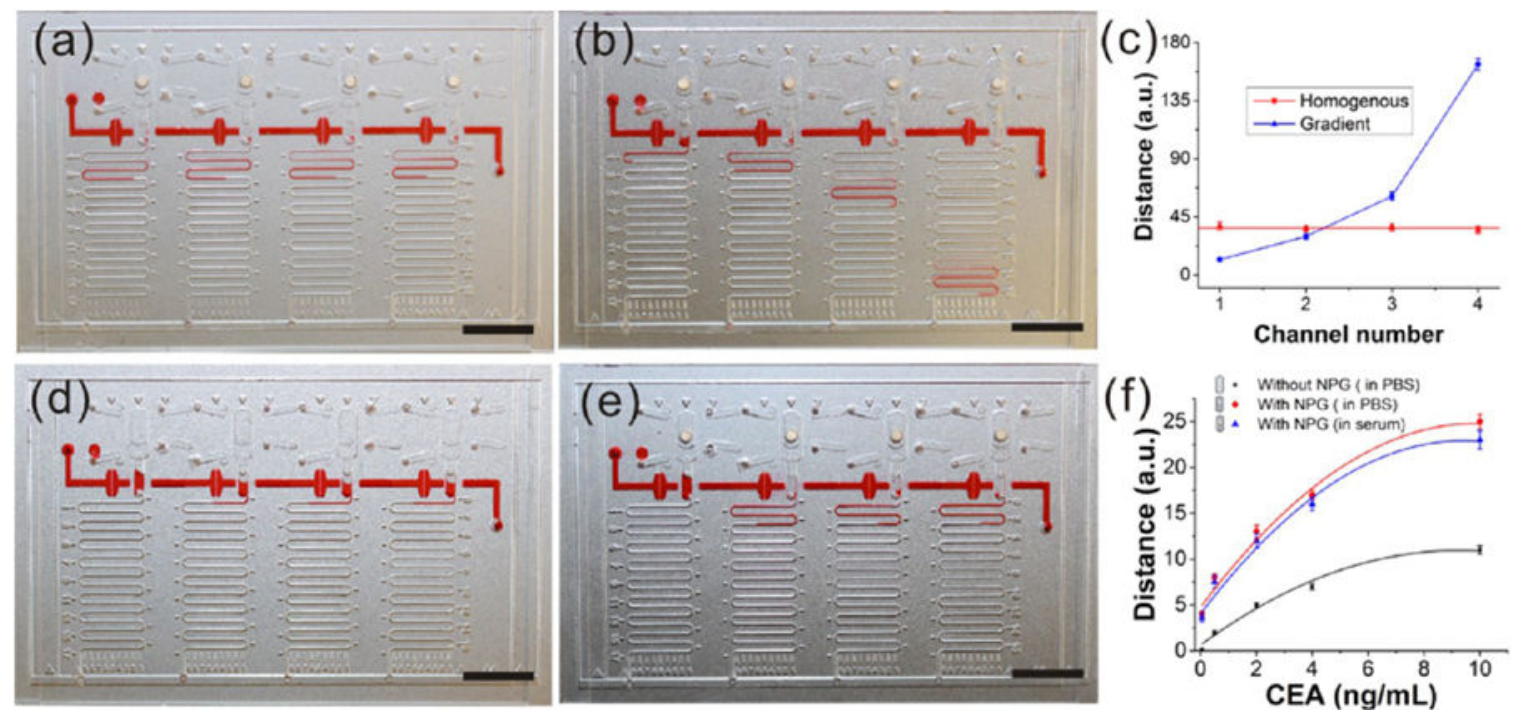

Figure 2.

Evaluation of the NPG-V-Chip. (a) Results of test using standard PtNP solutions of same concentration $(5 \mu \mathrm{g} / \mathrm{mL})$ in the four units. (b) Results of test with standard gradient PtNP solutions (from left to right: 2, 4, 10,50 $\mathrm{g} / \mathrm{mL}$ ). (c) Quantitation of bar-chart length for the homogeneous and gradient PtNP solutions using data obtained from (a) and (b). Bar-chart lengths were acquired from the rulers on the chip. Error bars were obtained from the results of three parallel experiments. (d and e) Results of CEA with concentration of $5 \mathrm{ng} / \mathrm{mL}$ on the device without (V-Chip, d) and with (NPG-V-Chip, e) NPG, respectively. (f) Results of assay of CEA in phosphate-buffered saline (PBS) or serum on the V-Chip (without NPG) and NPG-V-Chip (with NPG). Significantly greater ink advancement was observed in the NPG-V-Chip than on the V-Chip, demonstrating the higher sensitivity of the NGP-V-Chip. Serum substrate had a negligible effect on assay performance, compared with PBS. Barchart lengths were acquired from the rulers on the chip. Scale bar, $1 \mathrm{~cm}$ for (a), (b), (d), and (e). 
(a)

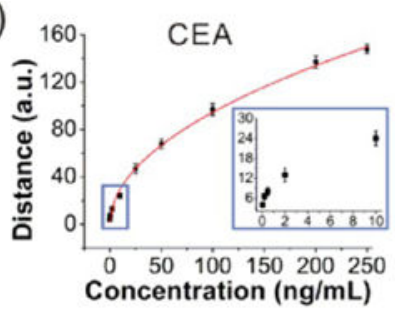

(d)

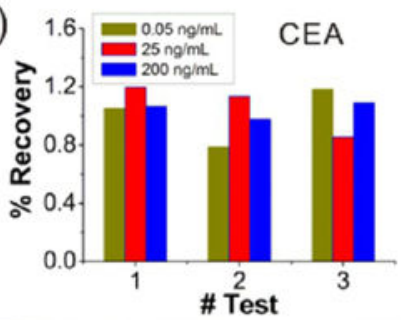

(g).

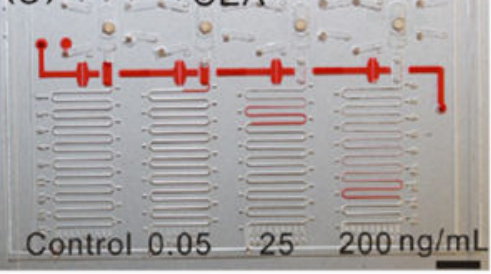

(b)

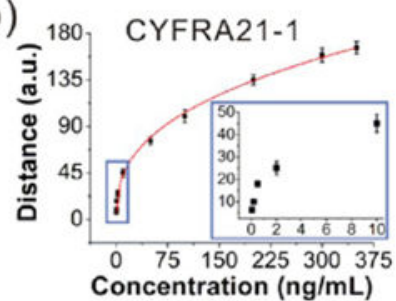

(e)

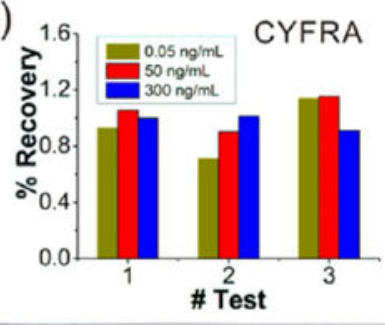

(h)

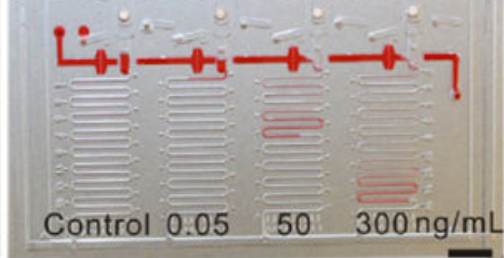

(c)

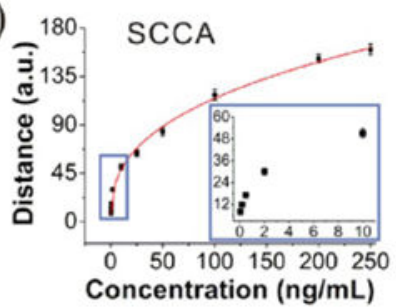

(f)
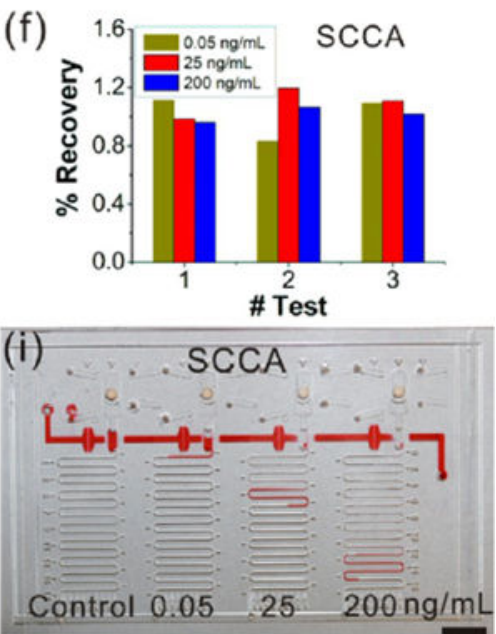

Figure 3.

Calibration curves for the three biomarkers of NSCLC. (a-c) Full response curves for CEA (a), CYFRA 21-1 (b), and SCCA (c). Insets show details for the indicated (boxed) lowconcentration standards at higher magnification. Error bars were obtained from the results of three parallel experiments. (d-f) Recovery for the indicated typical concentrations of CEA (d), CYFRA 21-1 (e), and SCCA (f). (g-i) On-chip bar-chart results for the indicated concentrations of CEA (g), CYFRA 21-1 (h), and SCCA (i). Scale bar, $1 \mathrm{~cm}$ for (g-i). 
(a).

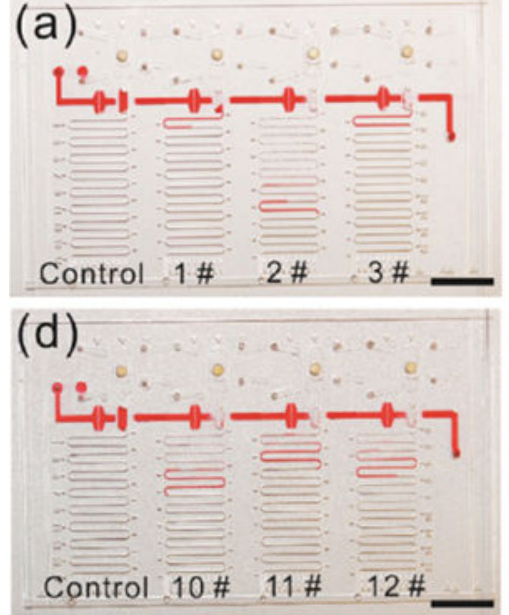

(g).

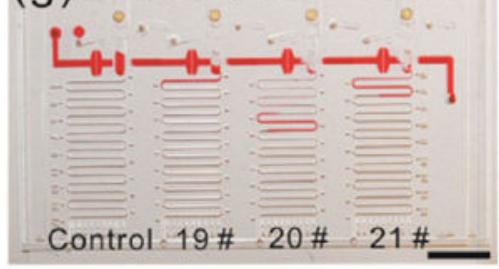

(b)

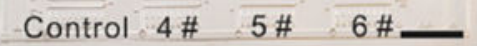

(e)

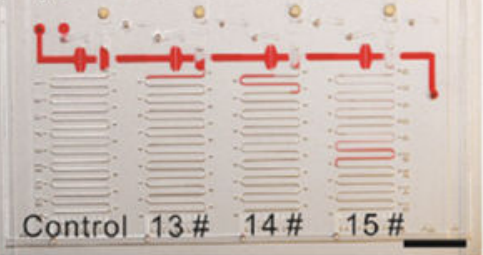

(h)

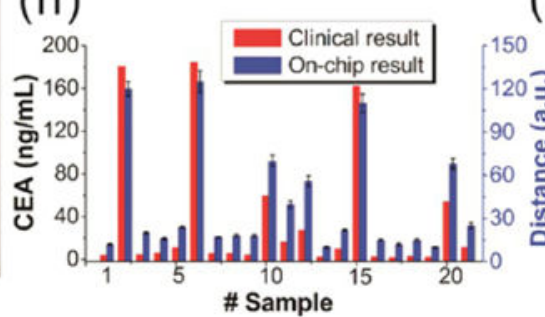

(c)

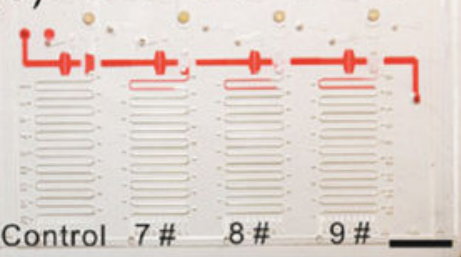

(f)
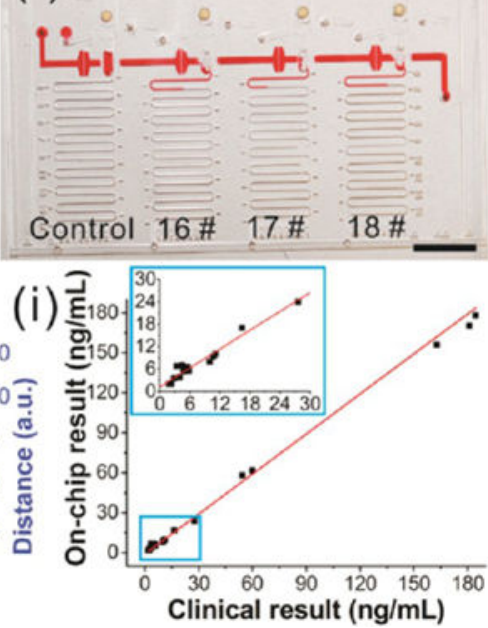

Figure 4.

Results of detection of CEA in serum samples of 21 patients with common cancers. (a-g) Bar-chart results for the 21 samples. Each panel shows a single test, which analyzes three samples. (h) Bar graphs of the CEA concentration determined by clinical assay (red) and NPG-V-Chip (blue). The error bars represent the SD of three parallel on-chip measurements. (i) Correlation of clinical and on-chip results. The result shows strong correlation between the two methods (slope $=1, R^{2}=0.99634$ for the linear fitting). Insets show details for the indicated low-concentration standards at higher magnification. Scale bar, $1 \mathrm{~cm}$ for $(\mathrm{a}-\mathrm{g})$. 
(a)
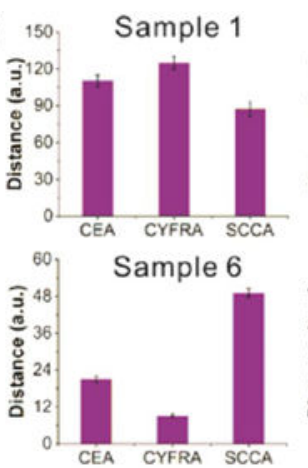

(b)

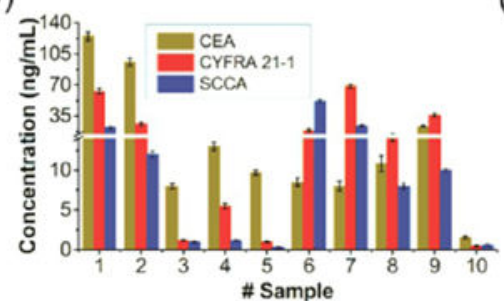

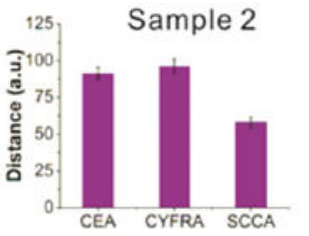

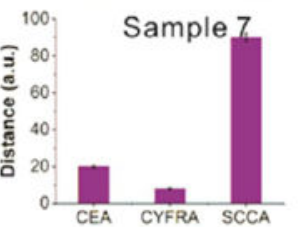

(c)
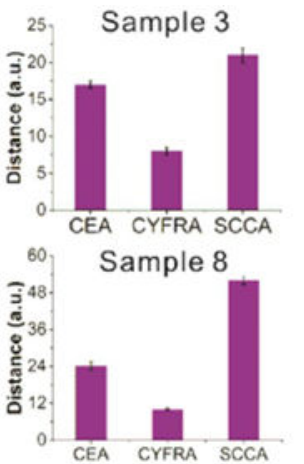
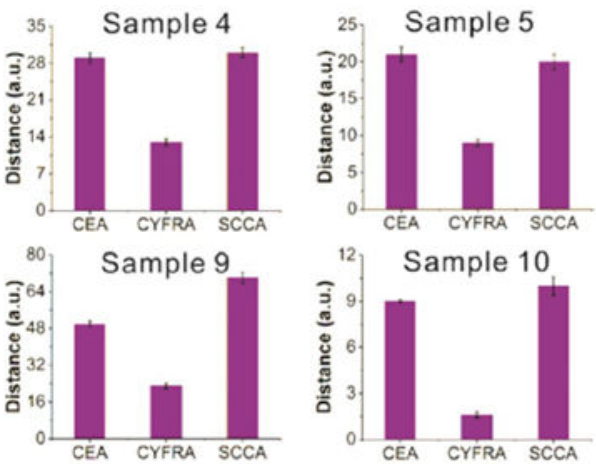
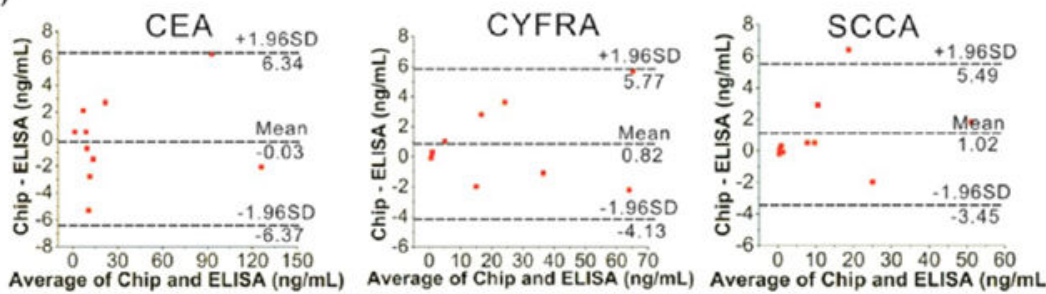

Figure 5.

Results of assay of the indicated biomarkers in 10 lung cancer patient samples. (a) Quantification of the on-chip results for the 10 individual samples. Data are relevant to that shown in Figure S6. The error bars represent the SD of three parallel on-chip measurements. (b) Quantitation of the concentrations of the three biomarkers in the 10 samples. Results were obtained based on the on-chip bar-charts and calibration curves. (c) Bland-Altman analysis for assessing agreement between results from the NPG-V-Chip and conventional ELISA. The 95\% confidence interval on the mean value is displayed in the graph. These results showed good correlation between the two methods. 